\section{P554 ADHERENCE AND SEXUALLY TRANSMITTED INFECTIONS AMONG MSM RECEIVING CARE IN A COMMUNITY- BASED HIV PREP CLINIC IN THE DEEP SOUTH}

${ }^{1}$ Paul St Clair*, ${ }^{2}$ Andrew Westfall, ${ }^{2}$ Matt Gravett, ${ }^{3}$ Will Rainer, ${ }^{3}$ Karen Musgrove, ${ }^{3}$ Elise Hayes, ${ }^{3}$ Josh Hicks, ${ }^{3}$ Shana Brooke Wheeler, ${ }^{4}$ Christina Muzny, ${ }^{5}$ Ellen Eaton. ${ }^{1}$ University of Alabama at Birmingham School of Medicine, Birmingham, USA; ${ }^{2}$ University of Alabama at Birmingham, Birmingham, USA; ${ }^{3}$ Magic City Wellness Center/BAO, Birmingham, USA; ${ }^{4}$ Univ Alabama, Birmingham AL, USA; ${ }^{5}$ University of Alabama at Birmingham, Medicine, Birmingham, USA

10.1136/sextrans-2019-sti.628

Background We have previously identified PrEP adherence as a risk factor for STIs in an academically affiliated Deep South PrEP clinic. In this analysis of a community-based Deep South PrEP clinic, we hypothesized that PrEP adherence and high risk sexual behaviors would be associated with STI.

Methods This was a retrospective analysis of men who have sex with men (MSM) aged $\geq 18$ years receiving PrEP at a community-based clinic in Birmingham, AL from 2016 to 2018. HIV negative patients were included if they completed $\geq 1$ sexual behavior and PrEP adherence survey and were prescribed PrEP. Screening for gonorrhea, chlamydia, and syphilis and patient-reported outcomes (PROs) were performed at quarterly clinic visits. The outcome of interest was incident bacterial STI. We evaluated the association of sexual behavior and PrEP adherence PROs with STI using logistic regression.

Results Of 119 eligible participants, 48\% were $\geq 35$ years, $21 \%$ reported no condom use, and $75 \%$ reported $\geq 2$ sexual partners. Over the study period, 29 cases of STI were detected including 18 chlamydia, 10 gonorrhea and 1 syphilis case. However, 14 participants declined STI testing. Of 85 patients who completed $\geq 1$ PRO (initial visit plus follow-up), 74\% reported excellent PrEP adherence. By univariate and multivariable analysis neither condom use, number of sexual partners, or PrEP adherence were significantly associated with STIs, though consistent condom use approached significance.

Conclusion In a community-based PrEP clinic in the Deep South, adherence was high and high-risk sexual behaviors were common. Neither adherence nor sexual behaviors were associated with STIs. Although it did not meet statistical significance, there was a trend toward STI for those reporting condomless anal sex. Surprisingly, many patients declined recommended STI testing, leaving missing data and suggesting an overall lack of awareness of STI risk in this community. These results highlight the need for vigorous STI screening and education

Disclosure No significant relationships.

\section{P555 MONITORING HIV AND SYPHILIS CO-INFECTION AMONG MEN WHO HAVE SEX WITH MEN IN BRAZIL, 2009-2016}

\footnotetext{
${ }^{1}$ Mark Guimaraes*, ${ }^{2}$ Maria Veras, ${ }^{3}$ Inês Dourado, ${ }^{4}$ Raimunda Macena, ${ }^{5}$ Ana Brito, ${ }^{6}$ Gustavo Rocha, ${ }^{7}$ Ana Rita Motta-Castro, ${ }^{4}$ Ligia Kerr. ${ }^{1}$ Federal University of Minas Gerais, Belo Horizonte, Brazil; ${ }^{2}$ Faculdade de Ciências Médicas da Santa Casa de São Paulo, São Paulo, Brazil; '3niversidade Federal da Bahia, Instituto De Saúde Coletiva, Salvador, Brazil; ${ }^{4}$ Federal University of Ceará, Fortaleza, Brazil; ${ }^{5}$ Centro de Pesquisas Aggeu Magalhães, Recife, Brazil; ${ }^{6}$ Federal University of São João Del Rei, Divinópolis, Brazil; ${ }^{7}$ Universidade Federal de Mato Grosso, Campo Grande, Brazil
}

10.1136/sextrans-2019-sti.629
Background HIV and Syphilis, including co-infection, are increasing among men who have sex with men (MSM) in several countries. The objective of this analysis is to compare the prevalence of HIV-syphilis co-infection, HIV only, or syphilis only among MSM in Brazil in 2009 and 2016 and and associated factors for 2016.

Methods Two MSM Respondent Driven Sampling (RDS) crosssectional studies were carried out in 2009 (10 cities) and 2016 (12 cities) in Brazil. HIV and syphilis serology were performed using standard rapid tests.Risk behavior and sociodemographic data were obtained. RDS weighted prevalence rates with $95 \%$ confidence intervals $(95 \% \mathrm{CI})$ were estimated. Poisson regression was used to estimate the prevalence rate ratio (PRR) and 95\%CI of each outcome, as compared to those with no infection.

Results The prevalence rates of syphilis only ( $80 \%$ increase) and HIV-syphilis co-infection ( $136 \%$ increase) were significantly different between 2009 and 2016 (Table 1). HIV only was relatively stable. For 2016 data, older age $(25+$ y.o.) and previous diagnosis of sexually transmitted infections were independently associated with co-infection, $\quad \mathrm{PRR}=2.49 \quad(95 \%$ $\mathrm{CI}=1.87-3.31)$ and $5.50 \quad(95 \% \mathrm{CI}=4.31-7.02)$, respectively. Other predictors were heterogenous with regard to different outcomes.

Conclusion Although the prevalence rate of HIV infection alone did not increase so between 2009 and 2016, it is of extreme concern the increase in rates of syphilis, alone or as co-infection with HIV, among MSM in Brazil. Treatment and prevention efforts may not be reaching those at higher risk and may lack effectiveness. Syphilis may be an important drive of the persistence of the HIV epidemic among MSM in Brazil. Nationwide public health actions, including syphilis testing, treatment and reiterating consistent condom use, are urgently needed.

Disclosure No significant relationships.

\section{P556 HISTORY OF STI THERAPY AND WILLINGNESS OF PREP AMONG HIV-NEGATIVE MSM COHORT IN JAPAN}

${ }^{1}$ Misao Takano, ${ }^{2}$ Daisuke Mizushima, ${ }^{2}$ Kazuko Tanaka, ${ }^{2}$ Takahiro Aoki, ${ }^{2}$ Yasuaki Yanagawa, ${ }^{2}$ Haruka Uemura, ${ }^{2}$ Koji Watanabe, ${ }^{2}$ Yoshimi Kikuchi, ${ }^{2}$ Shinichi Oka. ${ }^{1}$ National Center for Global Health and Medicine, Tokyo, Japan; ${ }^{2}$ National Center for Global Health and Medicine, AIDS Clinical Center, Tokyo, Japan

\subsection{6/sextrans-2019-sti.630}

Background Pre-exposure prophylaxis (PrEP) is not yet approved and information of PrEP is still very limited in Japan. In such circumstances, we established an HIV-negative MSM cohort at National Center for Global Health and Medicine, Tokyo in 2017. This study aimed to explore correlation between histories of STI therapies and willingness of receiving PrEP in this cohort.

Methods Face-to-face interviews with a semi-structured standardized questionnaire was conducted. HIV incidence risk index for MSM was used to identify the risk of HIV acquisition and scores of 10 and over were defined as at the high risk. Willingness of PrEP was divided into 4 categories: "Yes definitely", "Yes probably", "No probably" and "No definitely". Histories of STI therapies within 1 year against at least one of followings; syphilis, gonorrhea, chlamydia, hepatitis A, B, 
and $\mathrm{C}$ virus infection, genital herpes, amoebiasis, giardiasis, and condyloma, were recorded.

Results A total of 230 MSM completed the interviews from November 2017 through October 2018. The median age was 35 years (range: 18-70). MSM at the high risk was found in 185 (80.4\%). MSM who knew PrEP was only 61.3\%. Positive willingness of PrEP was observed in 64.8\%. MSM who answered "No definitely" were 21 among 185 high risk MSM. Their main negative reasons were "I think my HIV acquisition risk is low" in $12 \mathrm{MSM}$ and "I don't like to take medicine" in 8 . Histories of STI therapy within 1 year were found in 81 MSM (35.2\%). Among them, 27 (33.3\%) had still the negative willingness. There was no correlation of the positive willingness and history of STIs $(p=0.66)$.

Conclusion Still one third of MSM in our cohort lacked the proper knowledge of PrEP. Especially it is important to allow themselves to assess their own risk of HIV infection.

Disclosure No significant relationships.

\section{P557 "FLUX NZ": AN ONLINE NATIONAL COHORT INVESTIGATING HIV, STI AND DRUG-RELATED PRACTICES AMONG NEW ZEALAND GAY AND BISEXUAL MEN}

${ }^{1}$ Peter Saxton*, ${ }^{2}$ Mohamed Hammoud, ${ }^{3}$ Samuel Andrews, ${ }^{4}$ David Newcombe, ${ }^{5}$ Anthony Walton, ${ }^{5}$ Seb Stewart, ${ }^{5}$ Ricky Te Akau, ${ }^{6}$ Mark Fisher, ${ }^{7}$ Kathryn Leafe, ${ }^{8}$ Carl Greenwood, ${ }^{9} \mathrm{~J}$ Green, ${ }^{2}$ Garrett Prestage. ${ }^{1}$ University of Auckland, School of Population Health, Auckland, New Zealand; ' University of New South Wales, Kirby Centre, Sydney, Australia; ${ }^{3}$ New Zealand Drug Foundation, Wellington, New Zealand; ${ }^{4}$ University of Auckland, Centre for Addiction Research (CFAR), Auckland, New Zealand; ${ }^{5}$ New Zealand AIDS Foundation, Auckland, New Zealand; ${ }^{6}$ Body Positive, Auckland, New Zealand; ${ }^{7}$ New Zealand Needle Exchange Programme, Christchurch, New Zealand; ${ }^{8}$ Drugs Health and Development Project, Wellington, New Zealand; ${ }^{9} T e$ Whariki Takapou, Waikato, New Zealand

\subsection{6/sextrans-2019-sti.631}

Background HIV pre-exposure prophylaxis (PrEP) and chemsex present both opportunities and challenges for sexual health improvement among gay and bisexual men (GBM). However, there is scant evidence to inform responses in New Zealand (NZ), notably prevalence, uptake and cessation of behaviours over time. We sought a better understanding by recruiting a diverse national cohort of GBM.

Methods Flux NZ is a national online prospective observational study investigating wellbeing among GBM in NZ. The protocol, questionnaire and data management system were based on a concurrent Australian study. Recruitment was predominantly via social media and community partners. Eligible participants were GBM living in NZ. We present baseline descriptions of PrEP, STI and illicit drug-related data among the cohort to date.

Results The 315 participants are broadly reflective of the target population $(10.8 \%$ identified as indigenous Maori, 59\% lived outside Auckland the largest city, mean age 33.6 years (SD 15.6), 8.3\% diagnosed HIV-positive). Among HIV-negative participants, 50\% had heard "a lot" and 44\% "a little" about PrEP; 18.2\% were currently using PrEP. 57\% of all participants had tested for STIs in the past 6 months; 7\%, 5.7\% and 3.2\% were diagnosed with gonorrhoea, chlamydia and syphilis respectively. Drug use in the past 6 months was $37.5 \%$ cannabis, $35.6 \%$ amyl, 17.5\% MDMA, 5.1\% methamphetamine, 4.8\% LSD,
4.4\% cocaine, 3.2\% GHB, 2.5\% amphetamine, 1.0\% ketamine, $0.3 \%$ synthetic cannabis, $0 \%$ mephedrone, $0 \%$ heroin, and $1.9 \%$ other hallucinogens/psychedelics. "Any" drug use was $57.8 \%$ in the past 6 months and $78.7 \%$ ever. Of those using "party drugs" (MDMA, amphetamine, cocaine, methamphetamine, GHB, ketamine, LSD) recently, 29.2\% had done so to enhance sex, the majority "once" (4.7\%) or "a few times" (15.1\%).

Conclusion The Flux online cohort design effectively recruits GBM in NZ. This will enable comparative research of the impact of HIV, STI and drug harm reduction policies in the two countries.

Disclosure No significant relationships.

\section{P558 SEXUAL HEALTH, SYNDEMICS AND ASSETS AMONG MEN WHO HAVE SEX WITH MEN: SECONDARY ANALYSIS OF MULTI-NATIONAL SURVEYS}

${ }^{1}$ Lisa Mcdaid, ${ }^{1}$ Paul Flowers, ${ }^{2}$ Olivier Ferlatte, ${ }^{3}$ Kareena Mcaloney-Kocaman, ${ }^{4}$ Mark Gilbert, ${ }^{3}$ Jamie Frankis. 'University of Glasgow, MRC/CSO Social and Public Health Sciences Unit, Glasgow, UK; ${ }^{2} B C$ Centre for Substance Use, Vancouver, Canada; ${ }^{3}$ Glasgow Caledonian University, Glasgow, UK; ${ }^{4} B C$ Centre for Disease Control, Clinical Prevention Services, Vancouver, Canada

\subsection{6/sextrans-2019-sti.632}

Background Globally, gay, bisexual and other men who have sex with men (GBMSM) experience an increased burden of poor sexual, mental and physical health. Syndemics theory provides a framework to understand these interrelated health problems and how to intervene.

Methods Comparative quantitative secondary analysis of syndemic-related data are presented from two international, online, cross-sectional surveys: SMMASH2 in Scotland, Wales, Northern Ireland and the Republic of Ireland $(\mathrm{N}=3220)$; and Sex Now in Canada $(\mathrm{N}=7872)$.

Results In both studies negative sexual, mental and physical health outcomes were clustered, providing evidence of the syndemic (SMMASH2 O/E Ratio=1.32, 95\% CI 1.25-1.40; Sex Now O/E Ratio=1.59, 95\% CI 1.45-1.73). There were differences between the studies in the variables that were associated with experience of the syndemic. In Sex Now we found experience of the syndemic was associated with worries about sexuality-related stigma (AOR 1.87, 95\% CI 1.23-1.54) and experience of discrimination $(\mathrm{AOR}=1.83$, 95\% CI 1.60 2.10). Equally, some community assets appeared to have a protective effect on the experience of syndemics. In Sex Now, aspirations were significant; e.g., being unlikely to achieve quality of life significantly associated with increased odds of experiencing the syndemic $(\mathrm{AOR}=1.89,95 \%$ CI 1.63-2.19), while measures of community engagement were not significant. In SMMASH2, sense of coherence - a measure of resilience - was significant (AOR $=0.98,95 \%$ CI 0.96-0.99), indicating that higher sense of coherence was associated with decreased odds of experiencing the syndemic.

Conclusion These results present an important step forward in our understanding of syndemics. They provide new insights into how to intervene to reduce the interrelated burden of poor sexual, mental and physical health among GBMSM and point to a theoretical mechanism through which assets-based approaches to health improvement could function.

Disclosure No significant relationships. 